INPLASY

PROTOCOL

To cite: Tian et al. Effect of medical grade honey on cesarean wound healing: A protocol for systematic review and meta-analysis. Inplasy protocol 2020120054. doi: 10.37766/inplasy2020.12.0054

Received: 09 December 2020

Published: 09 December 2020

Corresponding author: Wenwu Gui

guifazu43395877@126.com

Author Affiliation:

Assisted Reproduction

Department, The First

Affiliated Hospital of

Chongqing Medical University

Support: MRP (NO.

2019MSXMI01).

Review Stage at time of this submission: The review has not yet started.

Conflicts of interest:

None.

\section{Effect of medical grade honey on cesarean wound healing: A protocol for systematic review and meta- analysis}

Tian, YR'; Gui, WW2 Zheng, ZY33 Feng, Q4

Review question / Objective: To systematically evaluate the effects of medical grade honey on cesarean wound healing. Condition being studied: medical grade honey, cesarean, wound healing, protocol, systematic review, meta-analysis. Information sources: PubMed, Embase, Cochrane Library, Web of Science, China National Knowledge Infrastructure, China Science and Technology Journal Database, and Chinese Biomedical Literature Database will be searched from inception of databases to December 2020. Additional trials will be searched by reviewing the reference lists of the retrieved articles, conference proceedings, and gray literature. The detailed search strategy for PubMed is shown in Table 1. The similar search strategies will be used for other electronic databases.

INPLASY registration number: This protocol was registered with the International Platform of Registered Systematic Review and Meta-Analysis Protocols (INPLASY) on 09 December 2020 and was last updated on 09 December 2020 (registration number INPLASY2020120054).

\section{INTRODUCTION}

Review question / Objective: To systematically evaluate the effects of medical grade honey on cesarean wound healing.
Condition being studied: Medical grade honey, cesarean, wound healing, protocol, systematic review, meta-analysis 


\section{METHODS}

Participant or population: Participants who have undergone cesarean will be included.

Intervention: In the treatment group, patients were given medical grade honey.

Comparator: It the control group, patients were given conventional treatments, placebo therapy, or no treatment.

Study designs to be included: All RCTs of medical grade honey for cesarean wound healing will be included. Case reports, animal experiments, reviews and republished studies will be excluded.

Eligibility criteria: RCTs of medical grade honey for cesarean wound healing.

Information sources: PubMed, Embase, Cochrane Library, Web of Science, China National Knowledge Infrastructure, China Science and Technology Journal Database, and Chinese Biomedical Literature Database will be searched from inception of databases to December 2020. Additional trials will be searched by reviewing the reference lists of the retrieved articles, conference proceedings, and gray literature. The detailed search strategy for PubMed is shown in Table 1. The similar search strategies will be used for other electronic databases.

Main outcome(s): Healing rate and healing time will be designated as the primary outcomes.

Additional outcome(s): Patient satisfaction and side effects will be designated as the secondary outcome.

Quality assessment / Risk of bias analysis: Two reviewers will independently assess the risk of bias of included studies by Cochrane risk of bias assessment tool. Random sequence generation, allocation concealment, blinding of participants and personnel, blinding of outcome assessment, incomplete outcome data, selective reporting and other bias will be evaluated. Any disagreement will be resolved by the third reviewer.

Strategy of data synthesis: If the number of included studies for the primary outcomes is less than 5 , only qualitative description will be carried. Data synthesis will be performed using Review Manager 5.3 (The Cochrane Collaboration, Oxford, UK). Odds ratio will be used for dichotomous outcomes with $95 \%$ confidence interval. Mean difference or standardized mean difference will be used for continuous outcomes. 12 test will be used to identify heterogeneity. The 12 value $>50 \%$ means significant heterogeneity, and the random effects model will be used. The fixed effects model will be utilized if 12 value $\leq$ $50 \%$.

Subgroup analysis: Subgroup analysis will be performed to explore the possible causes of heterogeneity based on the difference in participant characteristics, interventions, controls, and outcome indicators. If significant heterogeneity still exists after subgroup analysis, metaanalysis will not be pooled, and qualitative description will be reported.

Sensibility analysis: Sensitivity analysis will be conducted to test the robustness and reliability of pooled results by excluding combined studies one by one to observe whether there is significant change in the comprehensive results.

Country(ies) involved: China.

Keywords: medical grade honey, cesarean, wound healing, protocol, systematic review, meta-analysis.

Contributions of each author:

Author 1 - Yanran Tian.

Author 2 - Wenwu Gui.

Author 3 - Zeyu Zheng.

Author 4 - Qiang Feng. 